\title{
THE ROLE OF WIND TUNNELS IN PREDICTING SOUNDING ROCKET AERODYNAMICS
}

M. Leroy Spearman

January 1983 


\section{SUMMARY}

The aerodynamic characteristics of sounding rockets, in some cases, may be adequately determined by various estimating procedures. However, the estimating procedures are inadequate for configurations involving mi smatched sections, exceptionally long bodies, unusual payload shapes, and protuberances. Characteristics related to separated flow, interference flow fields, and Reynolds number may also present unpredictable problems. Hence it appears that, for the foreseeable future, experimental wind tunnel studies will remain a necessary factor in predicting the aerodynamic characteristics of sounding rockets.

\section{INTRODUCTION}

Sounding rockets have achieved a high degree of mission success over the years. Part of this success is due to the use of highly reliable components and to a reasonably good understanding of the aerodynamic behavior of the systems. In many cases, the flight characteristics can be predicted with sufficient accuracy because of the relative simplicity of the configurations and generally undemanding flight trajectories. However, in some cases, vehicle or flight characteristics are such that the successful prediction of performance should not be expected and experimental windtunnel investigations are required. Such cases may result from: flow separation; flow-field interference; unusual vehicle shape or size; excessive deviations to high angular positions; Mach number induced effects; Reynolds number effects; effects of protuberances; etc. The purpose of the present paper is to illustrate the nature of some of these cases as determined from wind tunnel studies. Several investigations related to sounding rocket aerodynamics are reported in references 1 to 15 .

\section{SYMBOLS}

A cross-sectional area of main cylindrical body

$C_{1}$ rolling-moment coefficient, $\frac{\text { rolling moment }}{\text { qAd }}$

$\mathbf{C}_{\mathbf{m}}$

$\mathrm{C}_{N}$ pitching-moment coefficient, $\frac{\text { pitching moment }}{q A d}$ normal-force coefficient, $\frac{\text { normal force }}{q A}$

c.p. center of pressure

diameter of main cylindrical body 


$\begin{array}{ll}h & \text { altitude } \\ l & \text { body length } \\ M & \text { Mach number } \\ q & \text { dynamic pressure } \\ R & \text { Reynolds number } \\ X & \text { Tongitudinal distance aft of nose apex } \\ \alpha & \text { angle of attack, degrees } \\ \Phi & \text { roll angle, degrees } \\ \text { Model Components } \\ B\end{array}$

\section{DISCUSSION}

An example of flow-field induced effects is shown in figure 1 where the experimental and estimated pitching-moment variation with angle of attack is shown for a relatively long body-wing configuration at $M=2$ (ref. 1). In the normal low angle operating range, the estimated and experimental results are in good agreement. However, near $\propto=12^{\circ}$, a pitch-up tendency occurs that is not reflected by the estimated characteristics. This difference results from a combination of effects that include an increase in the effectiveness of the forebody in producing lift at higher angles of attack and the downstream interference effects of the forebody-induced flow field on the wing stabilizing surfaces, both of which contribute to a forward movement of center of pressure and a nose-up pitching tendency.

A Schlieren photograph (fig. 2) shows the wake induced by the forebody as it passes the region of the tails for a body-tail configuration. Some of the effects of the forebody-shed vortices are discussed in references 2 and 3 . This is a complex problem that is still of concern and investigations in this area continue.

The effects of Mach number and body length on the pitch characteristics are illustrated in figure 3 for an Arcas rocket with an $2 / d$ of 18.2 and 23.8 (ref. 4 ). The pitch-up break tends to become more severe for the larger $\imath / d$. The tendency worsens with an increase in Mach number because of an overall decrease in stability 1eve1. This decrease in stability comes from an increase in forebody-1ift effectiveness which causes a forward shift in c.p. over the Mach number range shown that amounts to about 20 percent of the body length for either body. This decrease in stability level with increasing Mach number accentuates the possibility of pitch-up occurring at low angles of attack at the higher Mach numbers. Of course, the stability level in flight is also dependent upon the variation of center of gravity with fuel consumption and, for this reason, a precise determination of the c.p. variation from wind-tunnel tests is important. Subsonic and transonic data for these configurations are presented in reference 3. 
A Tomahawk launch vehicle for which the payload section was varied so that $1 / d$ values of 22.7, 24.7, and 26.7 were obtained is shown in figure 4 (ref. 5). For one of these vehicles, the fin planform was varied in anticipation of the stability change due to body extension. The effect of body length with the smaller fin (fig. 5) again points out the drastic reduction in stability level and the increased possibility of pitch-up with increased body length and increasing Mach number. For the reference center of gravity used, all of the vehicles would probably be stable for normal flight trajectories at $M=2.3$. At $M=4.6$, only the shortest vehicle shows the possibility of maintaining stable flight and then only for angles of attack less than about 4 degrees. The effect of the larger fin planform with the $2 / d=24.7$ body (fig. 6) is to substantially increase the stability level even though this configuration may become marginal at $M=4.6$ or above. The increase in stability resulting from the larger fin occurs because of the increased span, increased area, and sweptback trailing edge. An accurate estimate of the fin effectiveness could not be made because of the amount of the fin subjected to boundary-layer and vortex flows, and wind-tunnel results were desirable to define the regions of marginal stability.

The effect of a forebody size and shape change for a "hammer-head" type configuration is shown in figure 7 (ref. 6). For these configurations, the nose-cone slope is constant but the length of the cone is varied so that the maximum diameters of the forebody are different. Shapes of this type are not amenable to estimating techniques because of the flow-field changes aft of the maximum diameter. As shown by the experimental variation of $C_{m}$ with $\propto$ for $M=4.6$, such a forebody change can be significant. For the case shown, the change from the small forebody to the large forebody results in a change from a highly stable linear moment variation to a neutrally stable condition with a destabilizing nonlinearity with increasing $\propto$. This substantial change in stability with the larger forebody results from a combination of increased forebody lift and an increase in the interference flow-field effects on the tail. In addition, it should be noted that the minimum drag was doubled by the larger forebody and, for a given boost motor, this could have a significant effect on the flight performance.

Some effects of fin geometry on the aerodynamics of an Argo D-4 rocket with a bulbous forebody are shown in figure 8 (ref. 7 ). Calculations of fin effectiveness for this rocket are complicated by the presence of the bulbous forebody. The original configuration, which used fin 1 , is clearly unsatisfactory for $M=2.96$. Modifications included fin 2, which kept the same basic planform as fin 1, but had an increased span and a decreased taper ratio so that the aspect ratio and the area were both increased. Although the fin effectiveness did increase, the rocket was still neutrally stable at low angles and became unstable at higher c's. Fin 3 maintained the same area as fin 2 but had a swept planform and a single-wedge airfoil section. (Fins 1 and 2 had flatplate sections with beveled leading and trailing edges.) Although fin 3 has the same area and a lower aspect ratio than fin 2, the change in fin geometry is such that the vehicle with fin 3 is stable to $\alpha=6^{\circ}$ where a pitch-up instability begins. Additional results for another bulbous-nose configuration are presented in reference 8.

It is also necessary to know the lateral characteristics of sounding rockets before the bounds of satisfactory fiight can be established and the determination of these characteristics generally requires wind-tunnel testing. An illustration of the induced roll and yaw characteristics is shown in figure 9 for the mid-body length, sma11-fin Tomahawk rocket shown in figure 4. These results (ref. 5) are sufficient to indicate that significant, and sometimes catastrophic, induced moments may occur if the pitch or yaw stability permits a excursions beyond about 6 degrees. 
Relatively small protuberances may also have a pronounced effect on sounding rocket aerodynamics. Figure 10 is an example which shows the effect of smali antennae, in either an aft or a forward position, on the roll variation with o for a Cajun rocket (ref. 9). The induced roll is fairly small for the aft position but becomes considerably larger for the forward position. This difference is caused by the change in the interference flow-field region at the tail fins. This kind of induced effect is also generally found to be a function of variables such as Mach number and roll angle; thus, a substantial amount of experimental data may be required in order to quantify the magnitude of the effects over a complete flight range. Test results for a spinning and nonspinning model of a Cajun rocket are presented in reference 10.

A technique that may be useful in inhibiting induced roll has been subjected to some preliminary wind-tunnel tests (ref. 11). This technique (fig. 11) utilizes bearing-mounted aft tails that permit the tail section to relocate when an asymmetric flow field is encountered. In effect, the tail section tends to align with the local stream direction thus relieving the side loads and imparting no rolling moment to the body. The results shown in figure 11 indicate that the technique offers some potential for alleviating the problem of induced roll for sounding rocket type vehicles.

An effect of Reynolds number on the pitch characteristics of a spherical payloadrocket motor combination at $M=1.9$ is illustrated in figure 12 (ref. 12). This payload is the type used to launch inflatable satellites such as Echo. The successful: launching of such a satellite depends upon many factors, one of which is information concerning the orientation of the vehicle just prior to satellite inflation. Windtunnel tests disclosed that changes in the flow pattern around the vehicle occurred with changes in Reynolds number (or altitude). These flow-field changes were such that the wake of the sphere impinged on the rocket motor at the lower altitude (higher $R$ ) but did not impinge at the higher altitude (lower $R$ ). As a result, an erratic difference in the variation of $C_{m}$ with $\propto$ occurred that could affect successful inflation of the payload (lower left, fig. 12). The addition of spoilers aft of the sphere and forward of the rocket established the same flow pattern for both altitudes and a constant, linear variation of $C_{m}$ with $\propto$ resulted.

One other type of launch vehicle that has required wind-tunnel testing is the multi-stage, multi-fin system. A four-stage vehicle of this type is illustrated in figure 13. No discussion of this type vehicle is included in the present paper but investigations reported in references 13 to 15 indicate the problems of concern that required wind-tunnel studies. These problems include: mismatched diameters between stages; mutual fin interference effects; fin alignment and orientation; body defiection between stages; boundary-layer growth; and stability changes that occur as stages are dropped.

\section{CONCLUDING REMARKS}

Estimating techniques for the determination of sounding rocket aerodynamics should be used, to the extent possible, as an expedient way to predict filight characteristics. However, there are some limitations to the estimating techniques that place constraints on the vehicle geometry that is applicable and restrict certain kinds of flow-field changes. Several examples have been shown in which it was necessary to obtain wind-tunnel data in order to surmount the deficiencies of estimation procedures. It appears that, for the foreseeable future, experimental results will remain a necessary extension to predicted results in the determination of sounding rocket aerodynamics. 


\section{REFERENCES}

1. Spearman, M. Leroy: Aerodynamic Characteristics in Pitch of a Series of Cruciform-Wing Missiles With Canard Controls at a Mach Number of 2.07. NASA TN D-839, 1963.

2. Raney, D. J.: Measurement of the Cross Flow Around an Inclined Body at a Mach Number of 1.91. Tech. Note No. Aero 2357, British R.A.E., 1955.

3. Ferris, James $C_{.}:$Static Stability Investigation of a Single-Stage Sounding Rocket at Mach Numbers from 0.60 to 1.20. NASA TN D-4013, 1967.

4. Babb, C. Donald; and Fuller, Dennis E.: Static Stability Investigation of a Sounding-Rocket Vehicle at Mach Numbers from 1.50 to 4.63. NASA TN D-4014, 1967.

5. Jerne11, Lloyd S.: Aerodynamic Characteristics of a $0.25-5$ cale Model of Tomahawk Launch Vehicle at Mach Numbers from 2.30 to 4.63. NASA TM X-1684, 1968.

6. Blair, A. B., Jr.; and Carmel, Melvin M.: Wind-Tunnel Investigation of Three Proposed Launch Vehicles at Mach. Numbers from 2.30 to 4.63. NASA TM X-1498, 1968.

7. Hayes, Clyde; and Corlett, William A.: Supersonic Aerodynamic Characteristics of a 1/6-Scale Model of the Final Two Stages of the Argo D-4 Four-Stage Rocket Vehicle. NASA TM X-1152, 1965.

8. McKinney, Royce L.: Supersonic Longitudinal Stability Characteristics of the Two Final Stages of a Four-Stage Launch Vehicle. NASA TN D-2190, 1964.

9. Jerne11, Lloyd S.: Antenna Effects on the Aerodynamic Characteristics of a 0.410-Scale Model of the Cajun Rocket at Mach Numbers from 2.30 to 4.63. NASA TM X-1771, 1969.

10. Falanga, Ralph A.: Supersonic Investigation of a Spinning and Nonspinning Model of a Cajun (or Apache) Rocket Vehicle With Roll-Control Tabs. NASA TN D-2576, 1965.

11. Blair, A. B., Jr.: Wind-Tunnel Investigation at Supersonic Speeds of a CanardControlled Missile With Fixed and Free-Rolling Tail Fins. NASA TP-1316, 1978.

12. Robinson, R. B.; and Morris, 0. A.: Aerodynamic Characteristics of a Model of an Inflatable-Sphere Launching Vehicle Under Simulated Conditions of Mach Number and Altitude. NASA TN D-640, 1960.

13. Robinson, R. B.: Effects of Body and Fin Deflections on the Aerodynamic Characteristics in Pitch of a 0.065-Scale Model of a Four-Stage Rocket Configuration at Mach Numbers of 1.41 and 1.82. NASA TN D-37, 1959.

14. Gregory, D. T.; and Carraway, A. B.: Investigation of the Static Longitudinal Stability and Roll Characteristics of a Three-Stage Missile Configuration at Mach Numbers From 1.77 to 2.87. NASA TM X-124, 1959. 
15. Hinson, W. F.; Langhans, R. A. ; and Fournier, R. H.: Aerodynamic Characteristics in Pitch of a 1/7-Scale Model of a Two- and Three-Stage Rocket

Configuration at Mach Numbers of 0.4 to 4.63. NASA TN D-5378, 1969. 


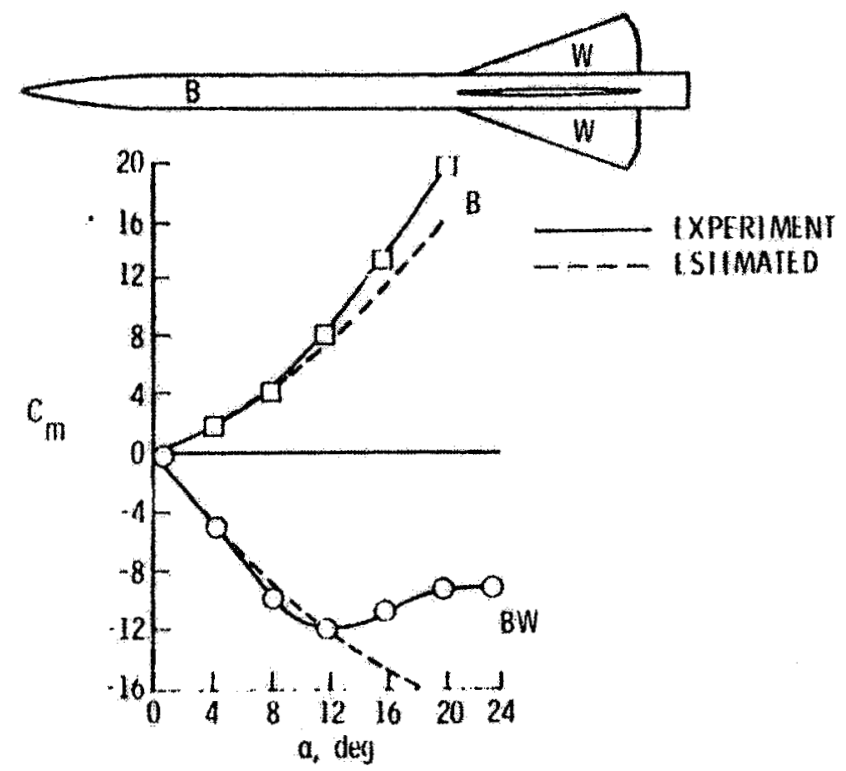

Fig. I Comparison of experimental and estimated results for a body-wing combination. $M=2, U d=19, \phi=0^{0}$.

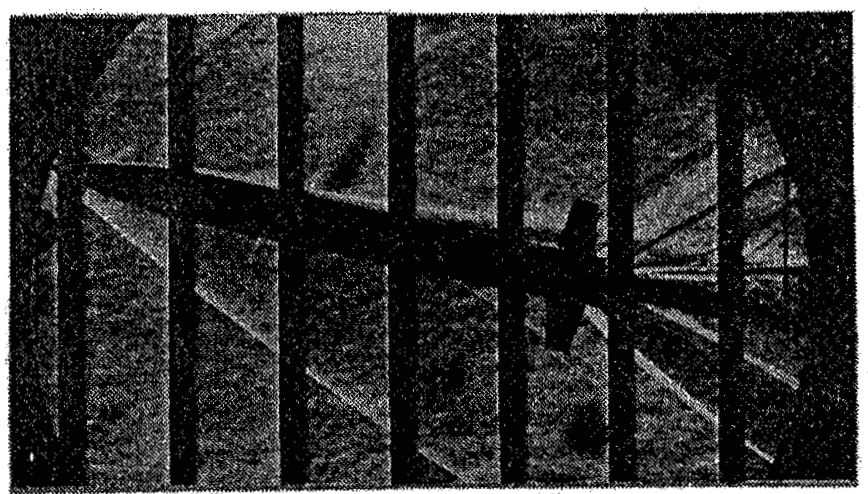

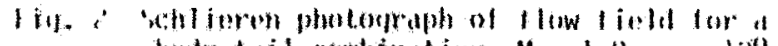

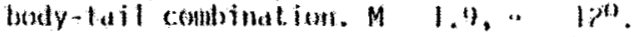



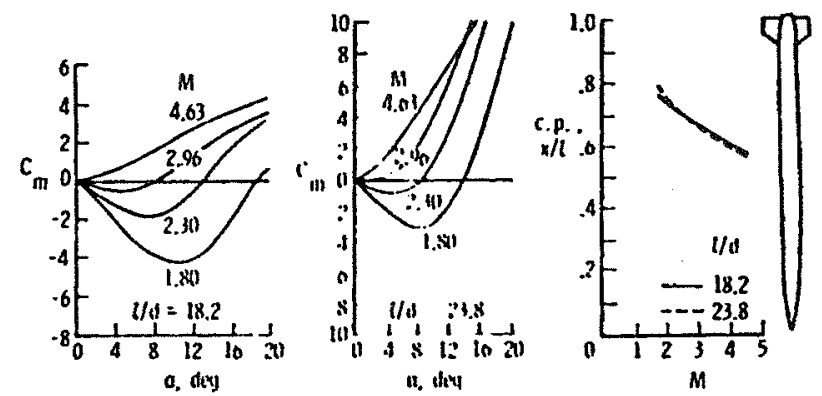

Fig. 3 Effect of Mach number and body length on pitching-moment characteristics.
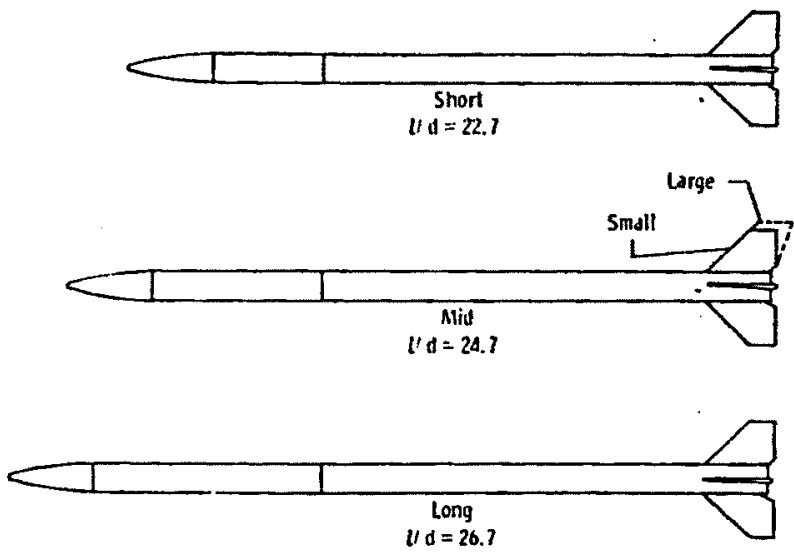

Figs 4 Geometric characteristics for a Tomahawk rocket.
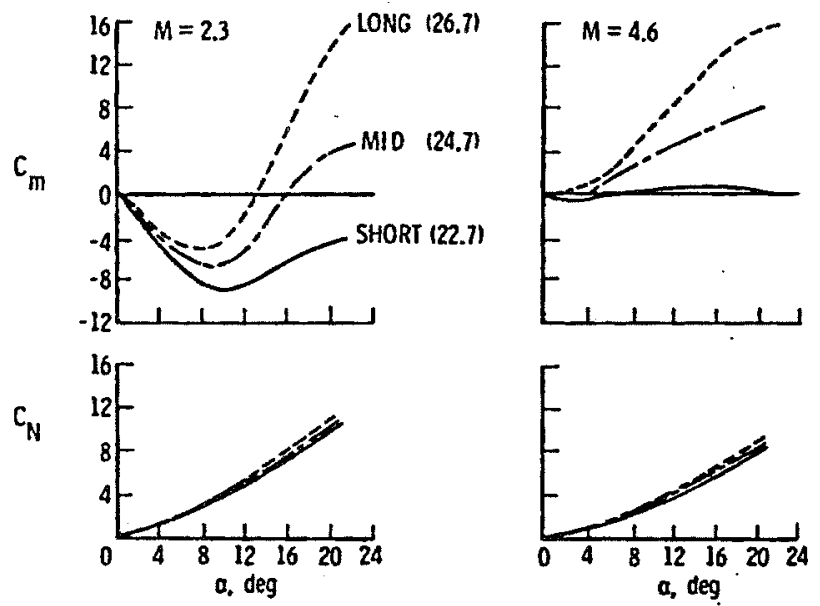

Fig. 5 Effect of body length on the nomal-force and pitching-moment characteristics of a Tomahawk with the small fin.
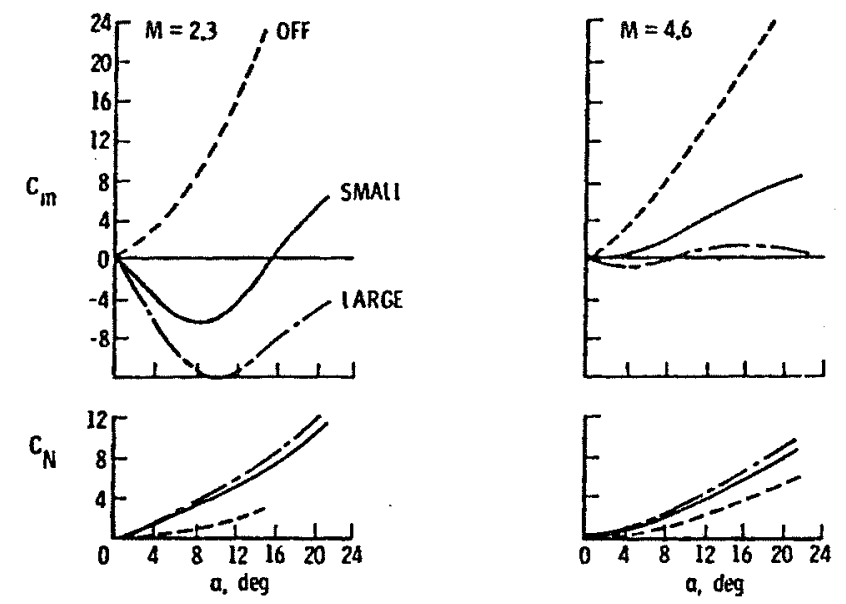

Fig. 6 Effect of fin planform on the nomal-force and pitching-moment characteristics of a Tomahawk. $\quad l / d=24.7$.
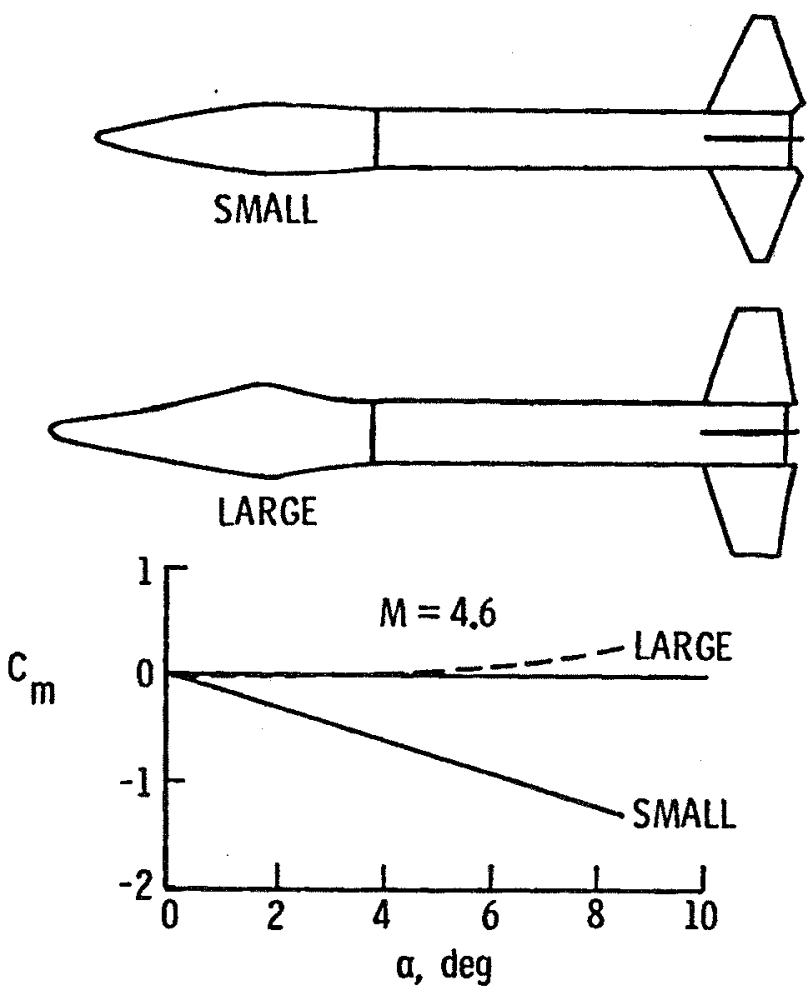

Fig. 7 Effect of forebody size and shape on pitching-moment characteristics. 

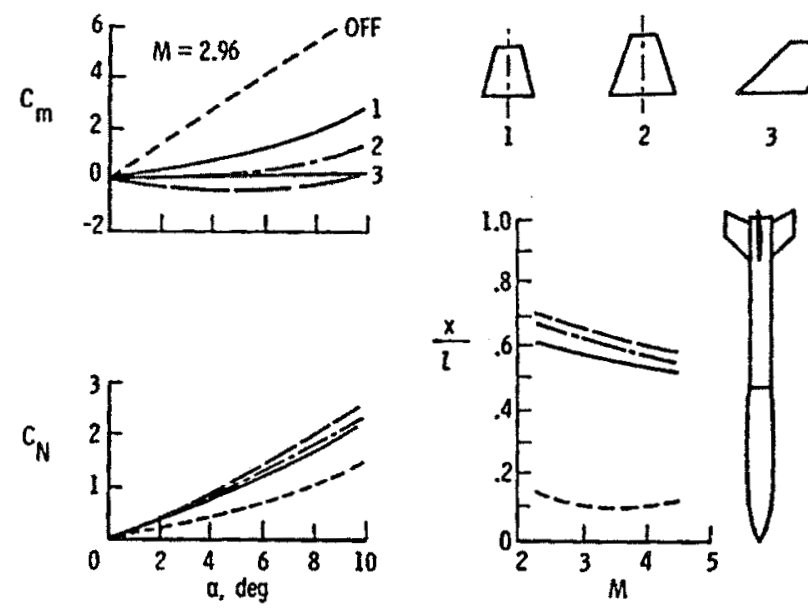

Fig. 8 Effect of fin geometry on the normal-force and pitching-moment characteristics of an . Argo D-4.
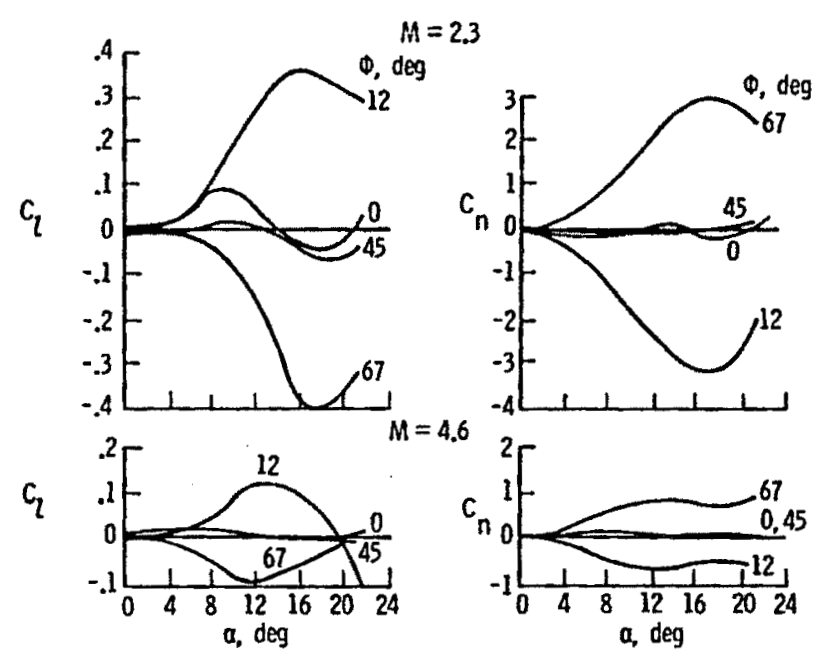

Fig. 9 Lateral characteristics for a Tomahawk with $i / d=24.7$ and the small fin.

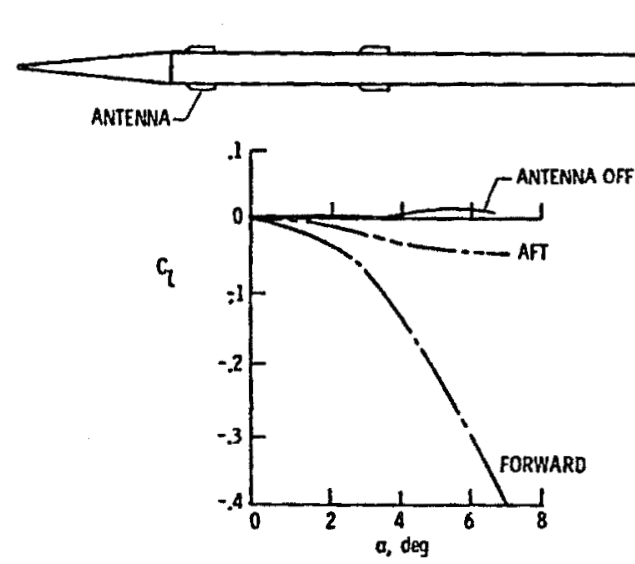

Fig. 10 Effect of antennas on the induced roll of a Cajun. $M=2.3, \Phi=45^{\circ}$.

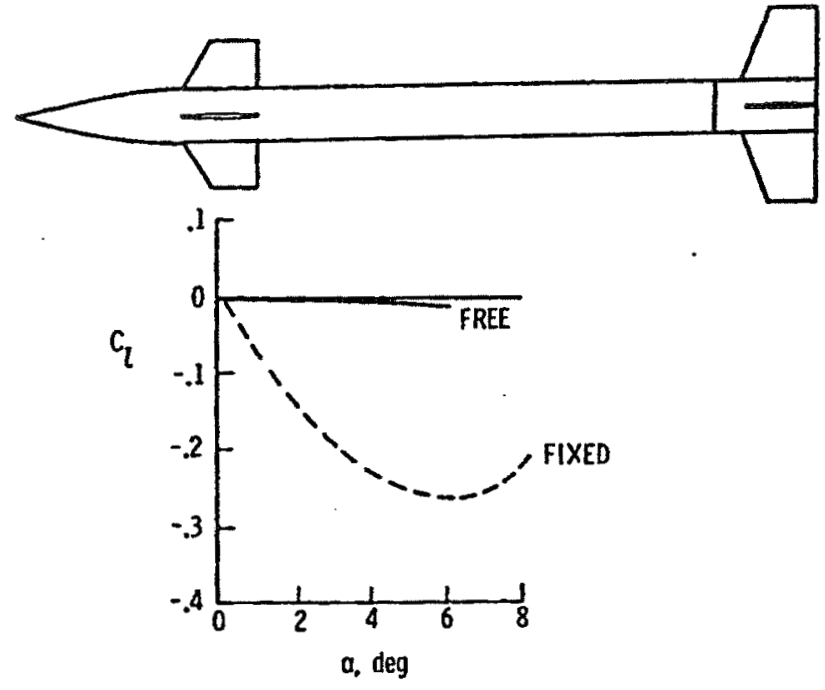

Fig. 11 Effect of a free-rolling tail on induced roll. $M=2.2$.
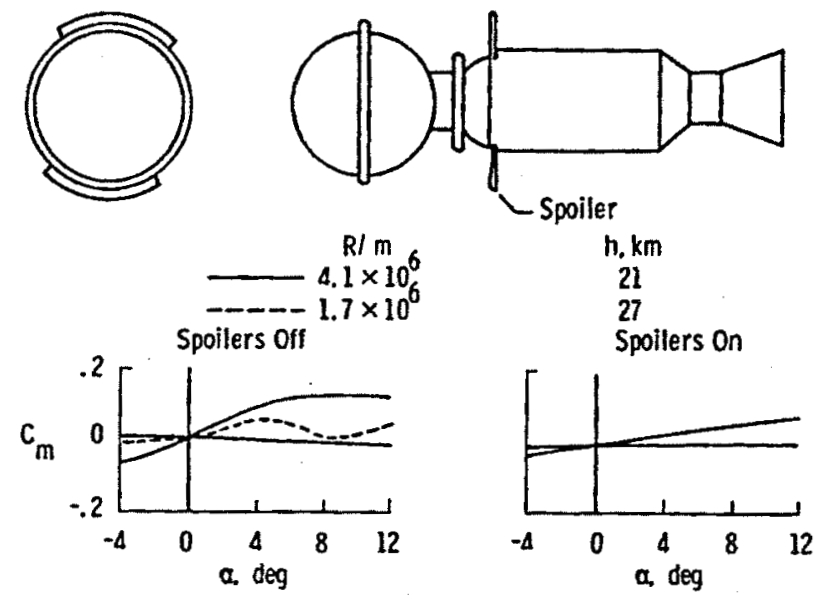

Fig. 12 Effect of Reynolds number on the pitchingmoment characteristics for a spherical payload - rocket motor combination. $M=1.9$.

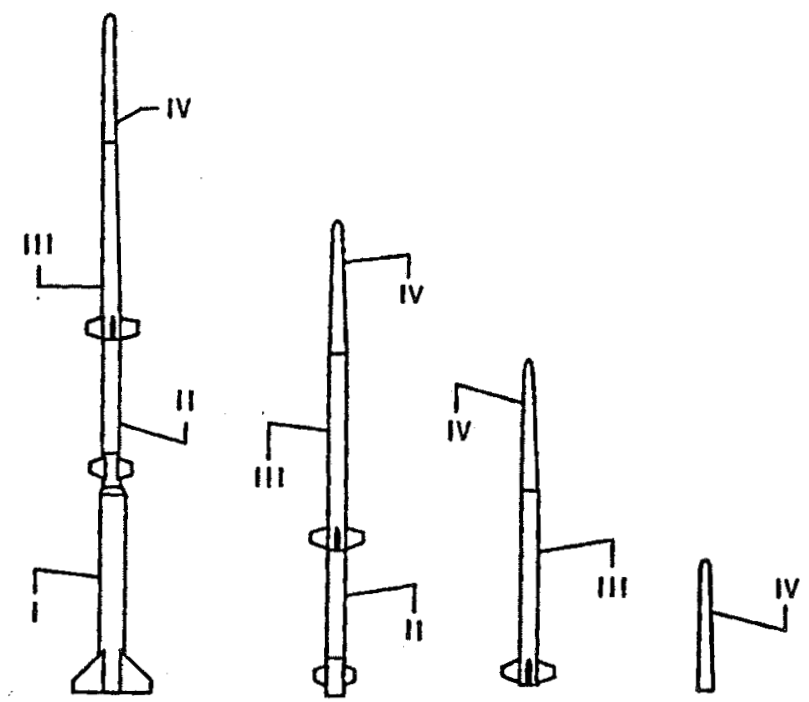

Fig. 13 Geometric characteristics for a four-stage sounding rocket. 


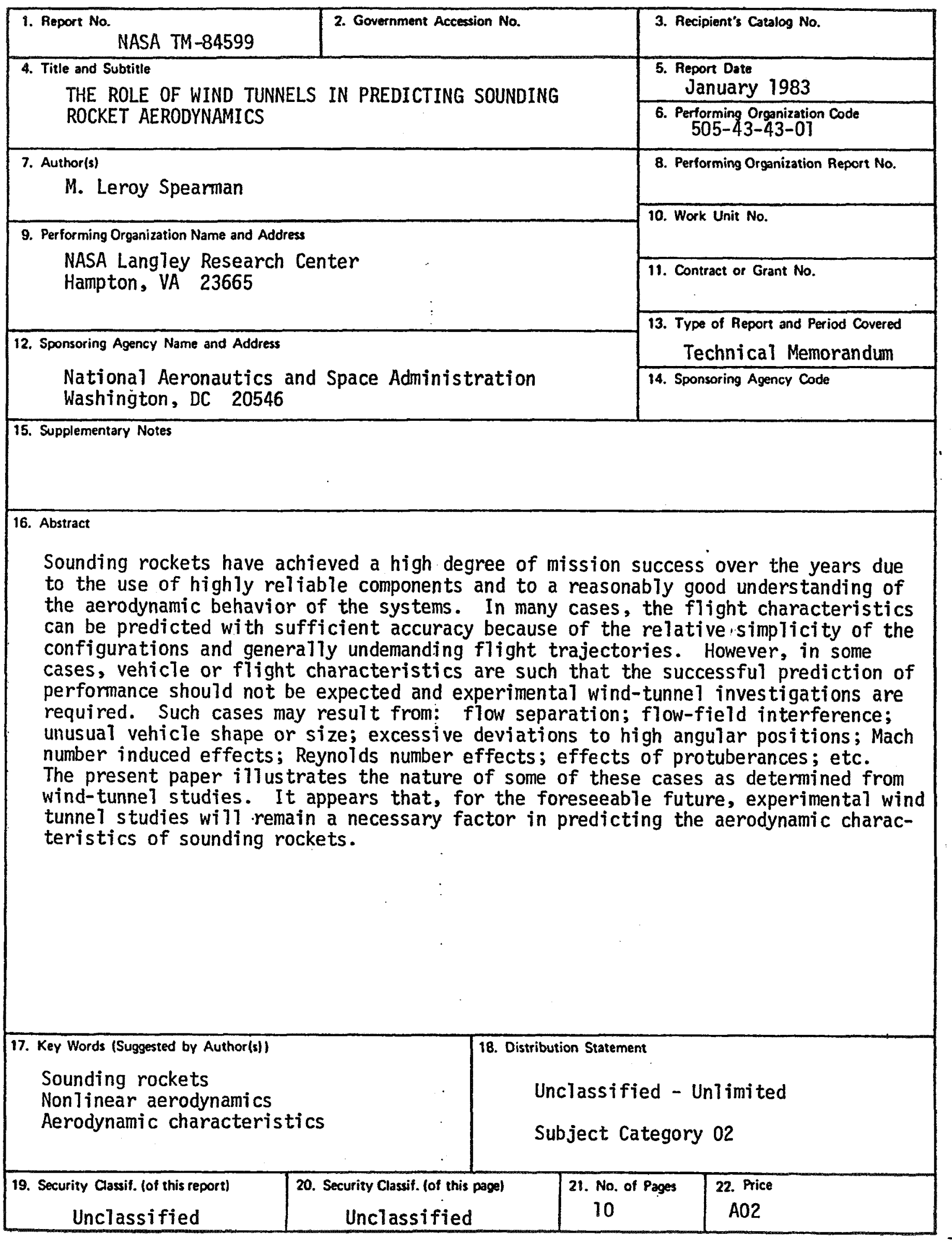

